\title{
L'ANALYSE MÉTAPHORIQUE D'UNE COMMUNICATION PROJECTIVE DANS LE CADRE DE L'APPROCHE CONSTRUCTIVISTE DE LA COMMUNICATION
}

\author{
Alex Mucchielli ${ }^{1}$
}

L'approche constructiviste de la communication a renouvelé l'interprétation possible du fonctionnement des techniques projectives. Cette nouvelle façon de concevoir la construction du récit projectif a amené avec elle un nouveau type d'analyse des récits obtenus. Cette analyse ne se fonde plus sur l'analyse de contenu et la recherche d'indices révélateurs de motivations ou de conflits intra-psychiques. Cette analyse est une analyse métaphorique dont la clef est donnée par la signification, implicitement partagée par un groupe culturel, au sujet de la planche projective utilisée. Je me propose de développer dans cet article ces différents éléments ainsi que les explications nécessaires à la démonstration de ces propositions.

1 Professeur à l'Université de Montpellier III.

Recherches en communication, $\mathrm{n}^{\circ} 2,(1994)$. 


\title{
La communication projective
}

Dans le cadre de référence existentiel (et non psychanalytique), la "projection" est l'expression du "monde intime" du sujet. Dès 1939, Franck montre l'existence, en chaque sujet humain, d'un "univers privé", monde intégralement subjectif de ses propres croyances. Ce monde privé oriente et informe activement les perceptions et les conduites ${ }^{1}$. Ce monde privé est une configuration particulière, constituée de nos "réactions affectives chroniques", ayant entre elles des réactions structurées, ce qui donne à cette configuration son style opératoire et ses effets singuliers. Pour Franck, on peut tenter la découverte de ce "processus dynamique" qu'est la personnalité en analysant ses "créations". Franck définit alors le concept de "méthode projective" pour rendre compte de la parenté entre le test d'association de mots de Jung (1904), celui de Rorschach (1921) et le T.A.T. de Murray (1935).

\begin{abstract}
"Dans son essence, dit-il, une technique projective est une méthode d'étude de la personnalité qui confronte le sujet avec une situation à laquelle il répondra selon le sens que la situation a pour lui, et suivant ce qu'il ressent au cours de cette réponse. Par suite, tout peut être utilisé comme technique projective, y compris les tests d'intelligence, à condition que l'examinateur considère par quel moyen idiomatique le sujet a répondu au test, au lieu d'utiliser les habituelles mesures normatives de comparaison... Le caractère essentiel d'une technique projective est qu'elle évoque du sujet ce qui est, de différentes manières, l'expression de son monde personnel et des processus de sa personnalité."
\end{abstract}

Le "monde personnel" d'un individu étant un monde subjectif de croyances, d'idées, de désirs, d'attentes... qui oriente et informe activement les perceptions et les conduites.

Cette conception est partagée par Bellak pour qui il faut considérer les images "projectives" présentées au sujet "comme des situations sociales déterminées" qui remplacent des situations réelles et qu'il faut considérer le comportement verbal des sujets devant ces planches comme l'expression de leurs sentiments profonds révélant "les structures actuelles de leur comportement social"2. Alors, nous dit-il,

1 L. K. FrancK, Projectives Methodes, Oxford, Buckwell Scientific Publ., 1948.

2 L. BELLAK, Guide pour l'utilisation du T.A.T., éd. du C.P.A., 1960, p. 5. 
interpréter les récits obtenus "signifie "découverte d'un dénominateur commun aux structures de comportement actuel d'une personne, et à la genèse de ces structures. Interprétation du T.A.T. signifie donc découverte de dénominateurs et de structures communs aux récits obtenus"'.

Bellak avait d'ailleurs mené des expériences à partir du T.A.T. pour mettre en évidence cette dernière conception de la projection. Après avoir recueilli les histoires imaginées par des sujets, Bellak les mettait en état d'hypnose et leur donnait l'ordre d'être agressifs puis, plus tard, l'ordre de se sentir déprimés et malheureux. Dans ces états, il recueillait leurs histoires imaginées aux planches du T.A.T. Dans le premier cas, il notait une forte augmentation des thèmes agressifs dans les récits; dans le deuxième cas, une forte augmentation des thèmes dépressifs. Bellak montrait ainsi que la projection est une intervention des prédispositions mentales du sujet dans les créations expressives de son imagination.

La communication projective est ce qui, dans nos expressions, notre discours... porte la marque de notre être propre, de quelque chose qui est profondément en nous, d'une sorte de "système de compétence"2 qui est à l'origine de tout ce que nous disons. Ce phénomène de "projection" de notre système interne dans nos expressions et nos conduites est un phénomène d'observation courante. Nous connaissons certains de nos amis qui sont préoccupés, voire "obnubilés", par quelque chose. On voit bien qu'ils ont tendance à tout ramener à leur problème, à tout voir d'un seul point de vue. Tels sont les pessimistes, qui voient tout en noir, interprètent tout dans le sens le plus négatif possible et nous laissent comprendre, finalement, qu'ils appréhendent le monde à l'aide de principes "excessifs" du genre: "rien de bon n'arrive", "tout va en se dégradant"... Leur état psychique interne déteint à la fois sur ce qu'ils perçoivent et sur ce qu'ils pensent et font. Tout ce qu'ils disent porte donc la marque de leur orientation d'esprit "pessimiste". "La communication projective c'est donc l'ensemble des choses que les hommes et les groupes disent sur leur façon de voir les choses, tout en communiquant sur autre chose qu'eux mêmes"3.

1 Ibidem, p. 7-8.

2 Le "système de compétence" est ce qu'un locuteur-auditeur possède en lui, qui lui permet spontanément de savoir si une phrase appartient à sa langue ou non (alors qu'il peut être dans l'incapacité de dire pourquoi).

3 Alex MuCCHIELLI, Psychologie de la communication, Paris, PUF, 1994, p. 143. 


\section{Le renouveau des techniques projectives à travers l'utilisa- tion du paradigme constructiviste des sciences de la communication}

Ce renouveau des techniques projectives a consisté d'abord à un retour aux sources en montrant comment Rorschach, lui-même, avait montré l'existence, dans les planches de son test projectif, d'un référent situationnel permettant aux sujets de construire, à partir de lui, leurs discours. Nous savons que le Rorschach est le prototype même de ce que l'on appelle un test projectif. Dans ce test, on montre successivement dix taches d'encre à un sujet et on lui demande de décrire tout ce qu'il voit dans les diverses figures. Dans son effort de construction de quelque chose de sensé à partir de taches informes, le sujet sollicite tous ses mécanismes perceptifs et donc tous ses processus psychologiques de compréhension du monde. Si l'on analyse l'ensemble des descriptions faites, on retrouve alors les traces de ces mécanismes psychologiques et le psychologue peut conclure à certaines particularités de la "structure de personnalité" du sujet.

Considérons la planche VI du Rorschach. On peut penser que cette planche ne représente rien, ni pour l'interviewer qui s'en sert, ni pour le sujet qui y réagit.

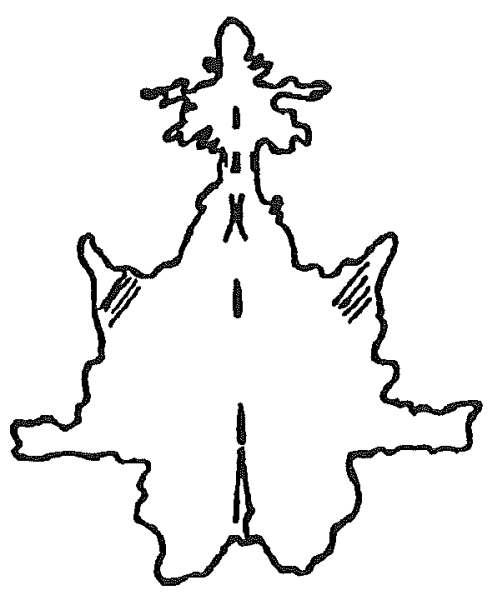

Schéma de la planche VI du test de Rorschach (P.U.F. éditeur)

A priori donc, la tache d'encre de la planche VI du Rorschach ne représente rien. Lorsque je réponds en inventant les choses que j'y 
vois, je fais œuvre de pure imagination. Mon imagination ne devrait pas être guidée par une problématique ou une question quelconque. Or, le remarquable de cette planche est qu'elle contient, à mon insu, un "référentiel implicite" par rapport auquel se fait ma réponsel. Examinons cela.

De très nombreuses expériences faites sur la planche VI du Rorschach ont montré que chaque fois qu'un sujet avait des problèmes sexuels, il y avait une perturbation de ses réponses à cette planche (et ce par rapport à son mode habituel de réponse aux autres planches). Tout se passait donc comme si la planche évoquait (inconsciemment) une situation sexuelle, situation qui elle, provoquait des réactions réelles pathologiques. Des vérifications ont été faites systématiquement auprès de sujets dont on connaissait, par ailleurs, les troubles du comportement sexuel. A chaque fois, ces sujets étaient "perturbés" devant la planche VI. Ainsi, par exemple, des recherches sur les jeunes garçons à la période pubertaire ont montré que ces jeunes étaient bloqués devant la planche VI ou la refusaient souvent. Or, on sait qu'à la période pubertaire, le jeune garçon découvre et doit gérer un ensemble de préoccupations nouvelles pour lui. C'est à la suite de ces expériences et constatations que l'on formula l'idée que cette planche, non figurative, représentait cependant "une situation de sollicitation sexuelle". Présenter cette planche revenait à évoquer cette situation pour le sujet.

La planche VI du Rorschach contient donc un référentiel caché mais cependant "aperçu" par tous les sujets. La planche VI du Rorschach et la situation de test où l'examinateur attend les commentaires du sujet sur la planche propose donc quelque chose à ce sujet. Planche et examinateur ne le laissent pas libre de "projeter" ce qu'il veut. Il lui est demandé de parler de ses réactions dans une situation de sollicitation sexuelle (et le sujet suit cette injonction). Du point de vue du paradigme constructiviste, on peut dire que dans la situation de test, le sujet examiné se plie à ce qui lui est demandé, la construction de sa réponse n'est pas uniquement laissée à ses mécanismes psychologiques, le thème de cette construction lui est donné. On constate donc que la présentation de cette planche VI revient à lui poser une question du genre: "comment réagissez-vous dans une situation de

\footnotetext{
1 Reprenant ces idées de Rorschach lui-même, les travaux de R. Mucchielli, $L a$ dynamique du Rorschach, Paris, PUF, 1968, ont démontré que c'était le cas pour toutes les planches du test.
} 
sollicitation sexuelle?". Dans la situation de test, la planche propose donc une problématique à laquelle répond le sujet. Les réponses données alors dans cette situation imaginaire, ont une analogie avec les réponses qui seraient données "en situation réelle".

A partir des éléments précédents, une série de recherches a permis de généraliser l'idée de référent culturel pouvant être contenu dans un matériel projectif figuratif. Par définition, le référent culturel d'une planche projective figurative est la situation implicite qu'elle représente pour un ensemble de sujets qui ont la caractéristique de "l'apercevoir" de la même manière". Ceci précise les conditions d'existence d'un tel matériel: il faut donc qu'il contienne une telle situation référentielle commune à toute une culture ou tout un groupe. De tels matériels existent, c'est en particulier le cas pour la très grande majorité des planches du célèbre test du T.A.T. de Murray². Je vais montrer ceci sur un exemple concret tiré des derniers travaux sur la communication projective ${ }^{3}$.

Considérons la planche ci-dessous ${ }^{4}$ :
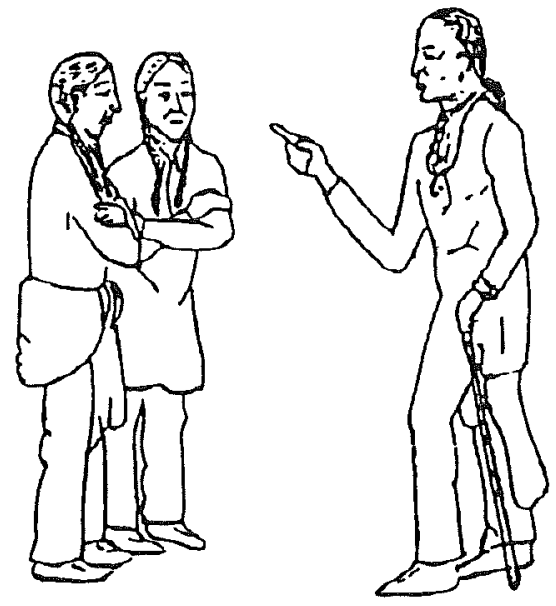

1 L'aperception étant la saisie immédiate et intuitive d'une forme et d'un sens.

2 Cf. sur ce point Alex MUCCHIELLI, L'analyse phénoménologique et structurale en sciences humaines, Paris, P.U.F., 1983, p. 181-182.

3 A. MuCChIELli, R. BREARD et P. PASTOR, L'entretien projectif, Paris, Eyrolles, 1991.

4 D'après W.E. HENRY, "Le T.A.T. dans l'étude des problèmes de groupe et des problèmes culturels, in H. ANDERSON et L. ANDERSON, Manuel des techniques projectives, Ed. Universitaires, 1965. 
Une banale analyse phénoménologique intuitive de cette planche nous montre que nous percevons immédiatement, et ensemble, une série d'éléments qui composent la structure de fond de cette figure. Ce qui fait la définition collective de la situation représentée par ce dessin, c'est la configuration des éléments qui ont chacun une définition culturelle claire (nous soulignons ces éléments ci-après). Remarquons que tous ces éléments nous sautent aux yeux en même temps. Nous les percevons ensemble -immédiatement et intuitivement. Ce n'est que par nécessité d'écriture que nous les décrivons, cidessous, un à un.

Les deux hommes plus jeunes sont côte à côte et se touchent même; cette proximité physique donne une indication sur leur relation "de personnes qui sont proches l'une de l'autre". Ils ont les bras croisés, ce qui immanquablement signale leur non activité, leur écoute ou leur attente. L'autre homme leur fait face et les regarde avec le doigt pointé-levé et est assez proche d'eux: ceci indique qu'il leur parle. Le doigt pointé et levé indique le conseil ou la mise en garde. Le personnage de droite s'appuie sur une canne. La canne est éminemment le signe de la vieillesse. Les physionomies indiquent des âges différents entre les deux personnages de gauche et celui de droite; ceci s'ajoute aux autres significations données par les autres éléments, confirme l'idée de situation où un plus âgé et plus puissant parle à des plus jeunes. Ceci prouve que les éléments constituant la "forme" de la situation (situation simulée ici) sont des abstraits déjà là, appartenant au cadre commun de référence. Ils fonctionnent en quelque sorte sous leur représentation conceptuelle. Ce sont les éléments: "plus jeunes", "plus âgé, canne", "regarde, doigt pointé", "bras croisés", "proche" et "face à face". Ensemble ces éléments construisent le sens de la situation. La situation est une situation de confrontation de jeunes à une autorité plus âgée. La planche contient cette problématique. Présentée à des "jeunes" comme à des "plus âgés", elle leur demande implicitement ce qu'ils pensent de cette confrontation.

Ainsi, la planche fait appel à une "situation idiomatique standard" anthropologique, c'est-à-dire, d' après Hall, une situation habituelle et très reconnaissable dans toutes les cultures. Cette situation, par ailleurs, appelle des "comportements idiomatiques standards", c'est-à-dire des réactions toutes préparées, régies par des normes sociales implicitement contenues dans la situation idiomatique évoquée par la planche projective, la compréhension du processus de construction de la réponse projective. Cette réponse va être une mise 
en scène personnelle faite par le sujet de la problématique posée par la planche. Autrement dit, le sujet va présenter, en l'interprétant à sa façon, cette problématique. C'est tout ce qu'il peut faire puisque la situation d'examen et la planche présentée l'oblige à répondre et à considérer la problématique implicite.

Ceci est démontré, en ce qui concerne les planches figuratives, par le fait que tous les récits projectifs faits à de telles planches (celles qui contiennent un référent culturel) font mention des éléments du "décor" que nous venons de signaler. Nous ne pouvons reprendre ici cette démonstration complète. Contentons nous de deux exemples.

\section{Récits obtenus à la planche de "l'autorité"}

Récit d'un adolescent de 15 ans:

"C'est deux jeunes, ils arrivent vers un homme, ils lui demandent, parce que l'homme il était riche et eux un peu pauvres, ils lui demandent des sous, pour gagner leur vie un peu; il leur donne des conseils, en même temps il leur demande de travailler chez lui, alors le couple ils ont accepté, ils ont fait tout ce qu'il fallait faire. Il leur a donné un peu de sous pour gagner leur vie, il leur donne du travail.

Un autre jour, ils sont revenus et l'homme était un peu plus sévère, alors il leur a dit: "Vous avez volé des bijoux de chez moi, je vous accepte plus, je vous emmène en prison".

Alors, les pauvres ils sont passés au tribunal et tout, ils voulaient se venger de lui parce que c'étaient pas eux qui avaient volé les bijoux; ils ont pris des années de prison, et quand ils sont sortis, ils ont trouvé une solution de se venger.

Ils l'ont invité chez eux, ils ont organisé quelque chose, alors l'homme riche il est venu, ils ont posé des trucs, ils ont pris des témoins exprès et tout, après ils l'ont fait disparaître. Il est passé au tribunal, mais tellement il était riche, il n'a pas pu rentrer en prison, alors l'homme du couple il est parti, et il a tué l'homme riche, il a organisé quelque chose et puis il l'a tué et voilà".

Récit d'une jeune fille de 19 ans:

Il n'y a pas si longtemps que ça, fin du XVIII $I^{\mathrm{e}}$ siècle; un grand seigneur vivait dans un immense château. Il avait deux fils qui étaient jumeaux, que devaient se partager l'héritage. Un jour leur père leur demanda d'aller chercher deux talismans dans un monde inconnu; ils étaient tout d'abord effrayés par cette idée, mais leur père leur dit que c'était le seul moyen de départager I'héritage: celui qui ramènera les deux talismans sera le bénéficiaire. Ils partirent chacun avec plusieurs équipements. 
Le premier, assez sérieux pensa à prendre tout ce qui concernait les boussoles, les cartes et l'autre beaucoup plus tête en l'air ne pensa qu'à prendre une couverture. Ils ne savaient pas exactement ce qu'ils devaient trouver, mais leur père leur avait donné un indice assez minime, c'était la couleur de cet objet qui était rare, de couleur ocre. Ils partirent sur le même chemin mais furent séparés par deux routes différentes, l'une menait vers la mer, l'autre vers la montagne.

Le premier parti vers la montagne car il avait les boussoles et les cartes et l'autre vers la mer. Son voyage vers la montagne fut assez périlleux, il rencontra des tas d'animaux sauvages, des bêtes féroces. Il chercha et pensa que cette couleur ocre ne pouvait venir que de la terre; il chercha à tout prix des grottes et marcha pendant des kilomètres. Il trouva une grotte et laissa tout son attirail dehors pour ne pas être gêné et se glissa dans la grotte; il parcourut quelques kilomètres et aperçut des paysages magnifiques. Il y avait du cristal dans tous les coins et de la couleur argentée, de l'or partout. Plus il avance dans la grotte, plus les couleurs deviennent ternes; quand il arrive au bout de la grotte, il trouve une pierre ocre tout à fait banale. Il eut l'impression que c'était le talisman et partit avec lui.

Par contre, son jeune frère, trop insouciant, continuait à marcher le long de la plage paisiblement tout en oubliant ce que lui avait demandé son père. Il avait un peu chaud et décida d'aller se baigner dans cette mer immense. Il fit quelques brasses et fut attiré comme un aimant par le fond; il se débattit et ne put rien faire; il arriva jusqu'à des profondeurs infernales. A ce moment-là, il perdit connaissance et lorsqu'il se réveilla, il trouva un nouveau monde sous la mer avec des poissons. Il les suivit comme hypnotisé et entra dans une grotte; il continua son chemin et trouva le même passage que son frère. Seulement, la différence, c'est qu'il fit le sens contraire de son frère: plus il avançait, plus les couleurs devenaient vives et belles. Il continua son chemin et au moment de sortir, il trébucha sur une pierre, il la regarda, la prit dans ses mains et fut attiré par elle; au moment où il la glisse dans son sac, il se transforme en pierre...

Dans ces deux exemples, nous trouvons effectivement la même situation de référence. Les récits font mention explicite de deux jeunes gens qui se trouvent face à une autorité. Mais nous pouvons apprécier la différence de représentation de cette autorité et de conduites face à elle. On voit comment l'imagination des sujets est libre d'inventer dans le cadre de la problématique proposée par la planche. 


\section{La lecture métaphorique des communications projectives obtenues à une planche à référentiel connu}

Dans l'analyse de contenu classique faite en termes de motivations, on recherche dans le contenu du discours développé, des thèmes précis, renvoyant à une liste de motivations. Le discours est donc décomposé en parties, chacune signifiant le passage à l'expression d'une motivation profonde, enfouie dans la structure du psychisme. Le discours n'est pas considéré comme une totalité de sens construite pour répondre à quelque chose de précis.

\begin{tabular}{|l|l|}
\hline \multicolumn{2}{|c|}{ Exemple d'analyse de contenu classique sur le premier récit } \\
\hline $\begin{array}{l}\text { C'est deux jeunes, ils arrivent vers } \\
\text { un homme, ils lui demandent, }\end{array}$ & Expression d'un besoin de contact \\
\hline $\begin{array}{l}\text { parce que l'homme il était riche et } \\
\text { eux un peu pauvres, ils lui demandent } \\
\text { des sous, pour gagner leur vie un peu }\end{array}$ & $\begin{array}{l}\text { Expression d'un besoin de } \\
\text { possession de richesses }\end{array}$ \\
\hline il leur donne des conseils..." & $\begin{array}{l}\text { Expression d'un besoin d'être } \\
\text { conseillé }\end{array}$ \\
\hline
\end{tabular}

Dans l'interprétation nouvelle du fonctionnement de la projection proposée à travers le paradigme constructiviste, tout le discours projectif (le récit) est considéré comme une totalité de sens. Son sens se découvre par la mise en rapport de sa totalité avec la situation de référence implicite contenue dans la planche. La découverte du sens n'est plus alors un exercice d'interprétation sans filet laissé à l'imaginaire de chaque psychologue (lequel puise d'ailleurs sa grille dans des théories fort diverses). La découverte du sens devient un exercice banal de lecture métaphorique. Ce qui est dit dans le récit projectif est une réponse à la problématique de la planche. Autrement dit, expliciter le sens du récit, c'est répondre à la question: "quelle réponse l'histoire développée apporte à la problématique de la planche?".

Ceci dit, en ce qui concerne les deux récits ci-dessus, le jeune garçon répond: "je considère que «l'autorité» est injuste et mauvaise. 
On peut essayer de s'en venger mais, de par sa puissance elle aura toujours le dessus. Le mieux est d'essayer de l'éliminer" et, la jeune fille répond: "je dois faire mes preuves par rapport à «l'autorité» qui veut me laisser un «héritage». Je crois que la meilleure façon de m'en sortir est d'être très sérieuse et organisée. Ma tendance à l'insouciance ne peut m'amener que des déboires".

Evidemment, de telles analyses peuvent être validées cliniquement a posteriori à partir d'un entretien avec ces sujets (et à partir d'une comparaison avec d'autres résultats obtenus à des tests déjà étalonnés). On fait normalement cette validation sur un certain nombre de sujets avant d'entériner la problématique d'une planche'1.

Les phénomènes que nous venons de décrire montrent que la planche projective est un partenaire à part entière dans la construction du monde que l'on fait à travers la communication qu'elle suscite. Ces recherches sur les techniques projectives dans le cadre du paradigme constructiviste des sciences de la communication montrent que les acteurs comme les éléments situationnels sont porteurs d'influences sur les communications. La planche proposée en situation de testage "oblige" le répondant à construire sa réponse sur l'orientation qui lui est donnée.

1 Cf. notre ouvrage sur "L'entretien projectif" pour voir aussi comment l'on peut construire des planches projectives qui abordent différentes problématiques. 